\title{
Million frames per second infrared imaging system
}

\author{
Alan T. Zehnder ${ }^{\text {a) }}$ \\ Department of Theoretical and Applied Mechanics, Cornell University, Ithaca, New York 14853 \\ Pradeep R. Guduru, Ares J. Rosakis, and G. Ravichandran \\ Graduate Aeronautical Laboratories, California Institute of Technology, Pasadena, California 91125
}

(Received 5 May 2000; accepted for publication 14 June 2000)

\begin{abstract}
An infrared imaging system has been developed for measuring the temperature increase during the dynamic deformation of materials. The system consists of an $8 \times 8 \mathrm{HgCdTe}$ focal plane array, each with its own preamplifier. Outputs from the 64 detector/preamplifiers are digitized using a row-parallel scheme. In this approach, all 64 signals are simultaneously acquired and held using a bank of track and hold amplifiers. An array of eight 8:1 multiplexers then routes the signals to eight $10 \mathrm{MHz}$ digitizers, acquiring data from each row of detectors in parallel. The maximum rate is one million frames per second. A fully reflective lens system was developed, consisting of two Schwarszchild objectives operating at infinite conjugation ratio. The ratio of the focal lengths of the objectives determines the lens magnification. The system has been used to image the distribution of temperature rise near the tip of a notch in a high strength steel sample (C-300) subjected to impact loading by a drop weight testing machine. The results show temperature rises at the crack tip up to around $70 \mathrm{~K}$. Localization of temperature, and hence, of deformation into " $U$ " shaped zones emanating from the notch tip is clearly seen, as is the onset of crack propagation. (C) 2000 American Institute of Physics. [S0034-6748(00)05710-5]
\end{abstract}

\section{INTRODUCTION}

Most of the energy that goes into permanently, or inelastically, deforming a material is dissipated thermally. Bending a paper clip back and forth until it breaks, and then putting the broken piece to one's lips to feel the temperature increase easily demonstrates this phenomenon. In many industrial processes and scientific problems involving dynamic, inelastic deformation of materials similar temperature increases occur. The increase in temperature can in some cases be very large in which case the temperature itself becomes an important parameter in the deformation process. Examples include dynamic fracture, ${ }^{1-3}$ metal cutting, ${ }^{4}$ formation of adiabatic shear bands, ${ }^{5,6}$ and ballistic impact. ${ }^{7}$ These processes all involve extensive permanent deformation occurring over a time scale that is too fast for the heat to dissipate to the environment before the deformation is complete. Large increases in temperature lead to thermal softening of the material, which promotes localization of deformation, and hence, even higher temperatures. High temperatures can also strongly affect the life of tooling used in manufacturing processes such as metal cutting. In other situations, such as impact at high, but not ballistic rates, or stable crack growth, there may be temperature increases moderate enough not to have a strong effect on deformation. Nonetheless, such temperature increases provide a useful means of gathering information about energy dissipation in the process.

In all of the examples described earlier it is desirable to measure the temperature of the object undergoing dynamic deformation. Ideally, the measurement should be noncontact, real-time, accurate, and spatially resolved. In the impact in-

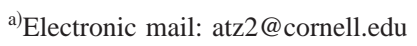

duced dynamic fracture and shear band problems we are interested in, deformation, fracture and the resulting temperature increases of $10-1000 \mathrm{~K}$ occur over time scales of a few tens to hundreds of microseconds and over size scales of $10-1000 \mu \mathrm{m}$. Thus, to study deformation induced heating and the resulting effects on the deformation process, a means of measuring temperature accurately, with high spatial resolution and at rates of around one million frames per second is required. Although there have been great advances in infrared (IR) imaging systems in recent years, there are no commercial systems capable of imaging at such high speeds. IR imaging systems typically run at television rates, $30 \mathrm{~Hz}$, although higher speed systems do exist, with rates of up to 34 $\mathrm{kHz}^{8}{ }^{8}$ Temperature at a point or averaged over a region can be measured at very high speeds using single element IR detectors in conjunction with high speed data acquisition. ${ }^{9,10}$ Linear arrays of up to 16 IR detectors have been used to measure the temperature field around dynamically growing cracks. ${ }^{1-3}$ Methods of measuring temperature during dynamic crack growth are reviewed in Ref. 3 .

Although a great deal can be done with single element and linear arrays of high speed IR detectors, to actually measure the temperature field requires a two-dimensional detector array. To achieve the required framing rates of up to 1 $\mathrm{MHz}$ requires very fast data acquisition in conjunction with a high-speed IR detector array. The bottleneck in performing such measurements has been data acquisition. However, the availability of fast, relatively inexpensive digitizers has now allowed us to design and build an IR imaging system that enables the measurement of thermal fields at rates of up to one million frames per second. The complete imaging system, consisting of the focusing optics, IR focal plane array, 


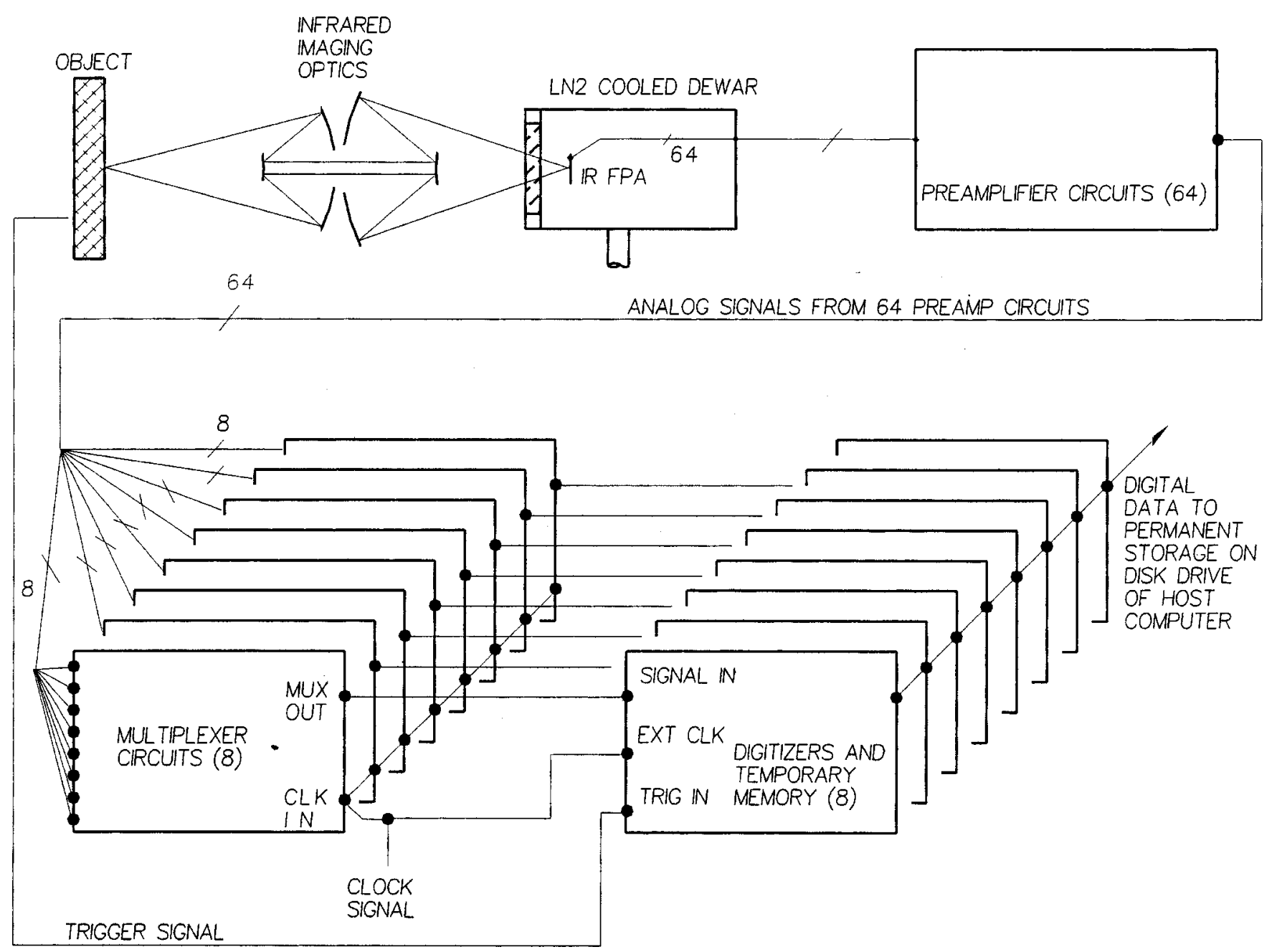

FIG. 1. Block diagram of million frames per second IR imaging system.

multiplexing circuits, and data acquisition boards is described in the block diagram of Fig. 1 and shown in the photograph of Fig. 2. At the heart of the system is an $8 \times 8$ focal plane array of $\mathrm{HgCdTe}$ IR detector elements. Each of the 64 elements has its own preamplifier, the outputs of which are fed into a bank of eight 8:1 multiplexers. The multiplexed signals are then digitized using 4 two-channel, Gage 1012 analog/digital (A/D) boards, running at speeds up

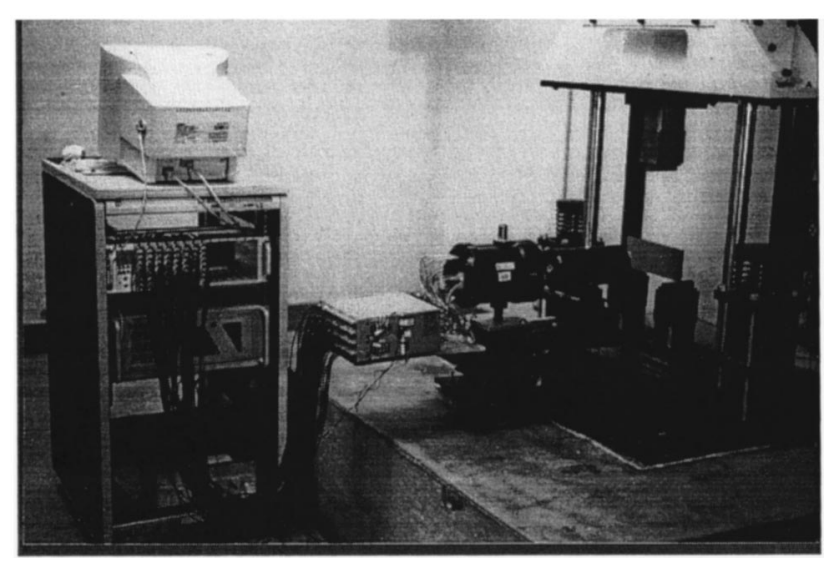

FIG. 2. Photograph of system in place in front of a cracked, steel sample to be loaded in impact at $6 \mathrm{~m} / \mathrm{s}$. to $10 \mathrm{MHz}$. Radiation emitted from the object as it deforms and heats up is focused onto the IR focal plane array using a reflective lens, built up out of two Schwarzschild objectives, each operating at infinite conjugation ratio. The magnification of each lens is fixed, thus to achieve different magnifications different lenses are used. In this system, there is no integration of the signal between frames as in commercial IR cameras; thermal resolution is sacrificed in favor of speed. The details of each of the components of the system are given in the next section, followed by examples of results obtained with the imager.

\section{SYSTEM DESIGN}

\section{A. Optics}

There are several requirements to be satisfied in the design of the optics. The setup of the system is greatly simplified if it can be focused using visible light. This leads to a fully reflective lens. The size of each IR detector element is $100 \times 100 \mu \mathrm{m}$. It was desired to have fields of view per detector ranging from approximately 180 to $50 \mu \mathrm{m}$. It is also important that the light gathering capability is large, i.e., low $f$ number, and that the blur at the focal plane due to various aberrations be less than $100 \mu \mathrm{m}$. Note that due to the rela- 


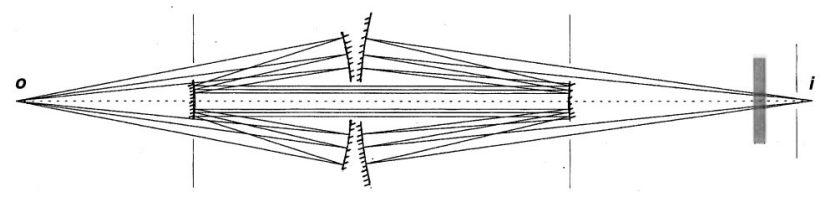

FIG. 3. Ray diagram of $1.3 \times$ reflective IR lens. The lens consists of two Schwarzschild objectives placed back to back, each used at infinite conjugation ratio. Magnification is given by the ratio of the focal lengths of the two objectives.

tively large size of the IR elements, achieving diffractionlimited performance in the optics was not necessary.

To obtain a range of magnifications, two lenses were designed and built, one with a magnification of $1.3 \times$ (or $1 / 1.3 \times$ if used in reverse) the other with $1.8 \times$ (or $1 / 1.8 \times$ ). Each lens consists of two Schwarzschild objectives, ${ }^{11}$ each operating at infinite conjugation ratio. Schwarzschild objectives consist of a large, concave primary mirror with a central aperture and a smaller, convex secondary mirror, located concentrically with the primary. The ratio of the primary mirror to secondary mirror focal lengths depends on the conjugation ratio used. At infinite conjugation ratio the best value is $f_{\text {primary }} / f_{\text {secondary }}=2.54 .{ }^{11}$ The $1.3 \times$ system is shown in Fig. 3. In the arrangement shown, the left side, or the object side, is a lens with $f=47.5 \mathrm{~mm}$. On the right side is a lens with $f=63.3 \mathrm{~mm}$. The ratio of the focal lengths of each side determines the magnification. Modules of this design are easily combined to achieve different magnifications. The lenses are located so that the focal point of the left side coincides with the object, the focal point of the right side coincides with the image.

For the actual lenses, rather than have custom lenses ground, we used gold-coated planoconvex and planoconcave mirrors from a standard catalog ${ }^{12}$ edged and drilled to our specifications. Thus, small deviations from the ideal $f_{\text {primary }} / f_{\text {secondary }}$ ratio of 2.54 were allowed in the interest of economy. The actual values range from 2.53 to 2.58 . We found by ray tracing that these deviations do not deteriorate the resolution greatly. For example, in the $1.3 \times$ system, both the left and right sides have a mirror ratio of 2.58. The spot size on the focal plane array (when used in the $1.3 \times$ magnification) is only $4 \mu \mathrm{m}$. The mirrors, housed in a black anodized aluminum cylinder are mounted ahead of the IR detector dewar as shown in the photograph of Fig. 2.

\section{B. Infrared detector array}

The temperatures of interest in dynamic deformation of materials range from $1-1000{ }^{\circ} \mathrm{C}$. At the lower range of temperatures, most of the radiation is near $10 \mu \mathrm{m}$. Thus, to achieve high sensitivity the IR detectors should have significant response at this wavelength. For this reason and for reasons having to do with availability of IR arrays and willingness of IR array manufacturers to build a custom array we chose to work with $\mathrm{HgCdTe}$ detectors. The IR detector array was custom built for this project. ${ }^{13}$ It consists of a back illuminated $8 \times 8$ array of $\mathrm{HgCdTe}$ elements. The elements are sensitive to radiation from approximately $2-10 \mu \mathrm{m}$, with the peak sensitivity at approximately $8 \mu \mathrm{m}$. The time response is very fast, limited only by the bandwidth of the preamplifiers.

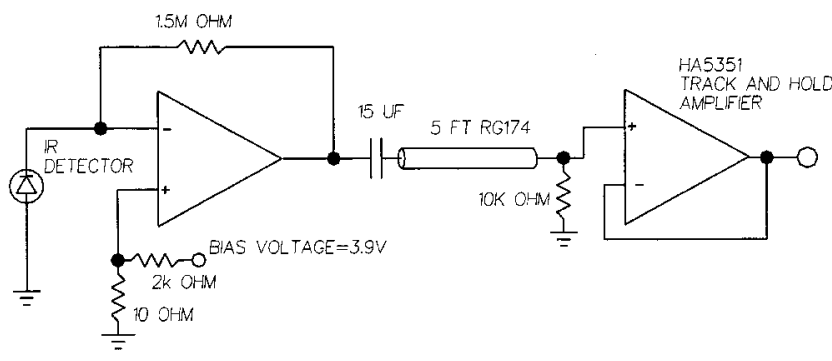

FIG. 4. Transimpedence circuit used to amplify current from the IR detector elements.

Each element is $100 \times 100 \mu \mathrm{m}$ with a center to center spacing of $130 \mu \mathrm{m}$. To minimize detector crosstalk, a ground plane surrounds each element. The array is indium bump bonded to a fan out board that mounts in a leadless chip carrier, housed in a side looking liquid nitrogen cooled Dewar. The detector elements are in vacuum, in contact with the liquid nitrogen cooled Dewar's cold finger and mounted behind a 6-mmthick $\mathrm{ZnSe}$ window.

The detector elements operate essentially as photodiodes; i.e., they output a current that is proportional to the incident radiation. This current is converted to voltage using the transimpedence amplifier shown in Fig. 4. The elements are held at a $3.9 \mathrm{~V}$ back bias, and the output is alternating current (ac) coupled to reject signals due to the detector's dark current. The bandwidth of the detector-amplifier system is $500 \mathrm{kHz}$.

\section{Multiplexers and data acquisition}

The key to acquiring images at the desired one million frames per second rate is fast, low noise data acquisition. One approach would be to have 64 digitizers, one for each pixel of the array. Such an approach is not only clumsy but was well beyond our budget. Thus, an alternative approach was taken using row-parallel data acquisition. In this scheme, each row of elements is fed into its own custom built 8:1 multiplexer. The output from each multiplexer is digitized by one of the eight $10 \mathrm{MHz}$ digitizers.

The block diagram of the multiplexer circuit is shown in Fig. 5. At the front end of each multiplexer are eight HA5351 track and hold amplifiers, configured for unity gain. The first four inputs are fed to one CLC533 4:1 multiplexer and the second four are fed to another CLC533. The outputs of these are fed to a third CLC533. When, for example, images are to be acquired at one million frames per second, a $10 \mathrm{MHz}$ clock is provided to the timing circuit. The timing diagram is shown in Fig. 6. The timing circuit is essentially a decade counter. It provides a track and hold signal to the HA5351 causing it to track the inputs for $100 \mathrm{~ns}$, then hold them for $900 \mathrm{~ns}$ while the multiplexers switch through the outputs ten times per frame, i.e., every 100 ns. After the eight input signals are switched through the system, a $0.5 \mathrm{~V}$ signal is fed to the output for $200 \mathrm{~ns}$. During the time the 0.5 $\mathrm{V}$ signal is sent to the output the track and hold amplifiers are tracking, then acquiring the signals. This provides sufficient tracking and settling time for the HA5351. The $0.5 \mathrm{~V}$ signal provides a marker in the output record signifying 
INPUT SIGNALS

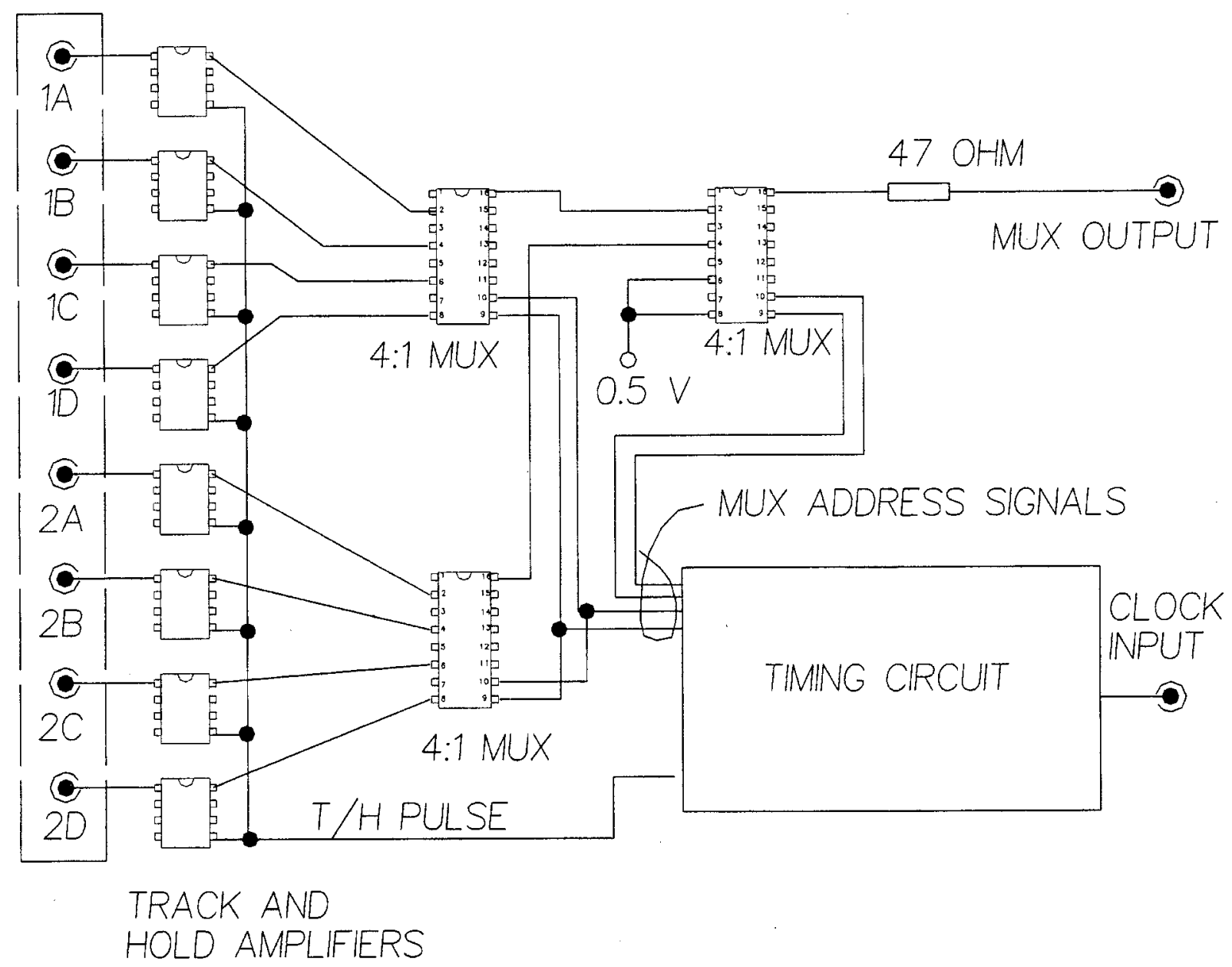

FIG. 5. Multiplexer block diagram.

where a frame begins and ends. With reference to Fig. 5, the sequence of outputs from the multiplexer is $1 \mathrm{~A}, 2 \mathrm{~A}, 1 \mathrm{~B}, 2 \mathrm{~B}$, $1 \mathrm{C}, 2 \mathrm{C}, 1 \mathrm{D}, 2 \mathrm{D}, 0.5 \mathrm{~V}, 0.5 \mathrm{~V}$.

The output signals from the eight multiplexers are fed to the inputs of four two-channel, $10 \mathrm{MHz}, 12$ bit A/D boards (Gage 1012) mounted in an industrial PC. Each board has $512 \mathrm{k}$ memory, sufficient to store up to 25000 frames of data. To ensure synchronization, the A/D boards are clocked externally using the same clock that drives the multiplexers. Data is acquired $40 \mathrm{~ns}$ after the rising edge of the clock. Thus (see the timing diagram of Fig. 6) data is acquired at approximately $40 \mathrm{~ns}$ after the multiplexers have switched from one output to the next. The settling time of the CLC533 multiplexers is about $25 \mathrm{~ns}$, so the $40 \mathrm{~ns}$ delay provides

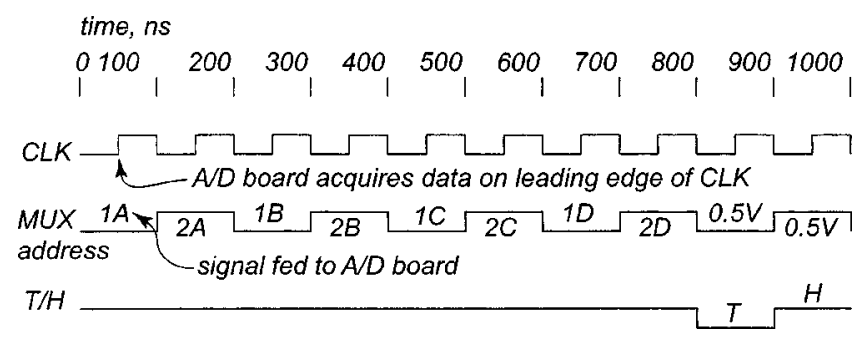

FIG. 6. Timing diagram for multiplexer and A/D board. sufficient time for the signal to settle before the data are digitized. Experiments are typically run in a single shot mode whereby a number of frames are acquired at high speed, stored on the memory of the A/D boards, and then transferred to the host computer's disk drive once the test is over.

\section{SYSTEM CALIBRATION AND TEST}

To ensure integrity of the results great care must be taken in calibrating the system, understanding the system limitations, noise levels, accuracy, precision, and both spatial and temporal resolution.

\section{A. Spatial resolution}

The spatial resolution is determined by the resolution of the optics, by the element size, and crosstalk of neighboring IR detector elements. The detector element, or pixel, size is $100 \mu \mathrm{m}$ square. The maximum magnification lens used is $1.8 \times$, thus the best attainable spatial resolution is $55 \mu \mathrm{m}$. Resolution could be increased using higher magnification lenses, however, since the radiation being imaged is in the range of $10 \mu \mathrm{m}$ wavelength, one cannot expect to do much better than about $20 \mu \mathrm{m}$ spatial resolution. To test the actual resolution a vertically oriented $75 \mu \mathrm{m}$ diameter, $\mathrm{NiCr}$ wire is 


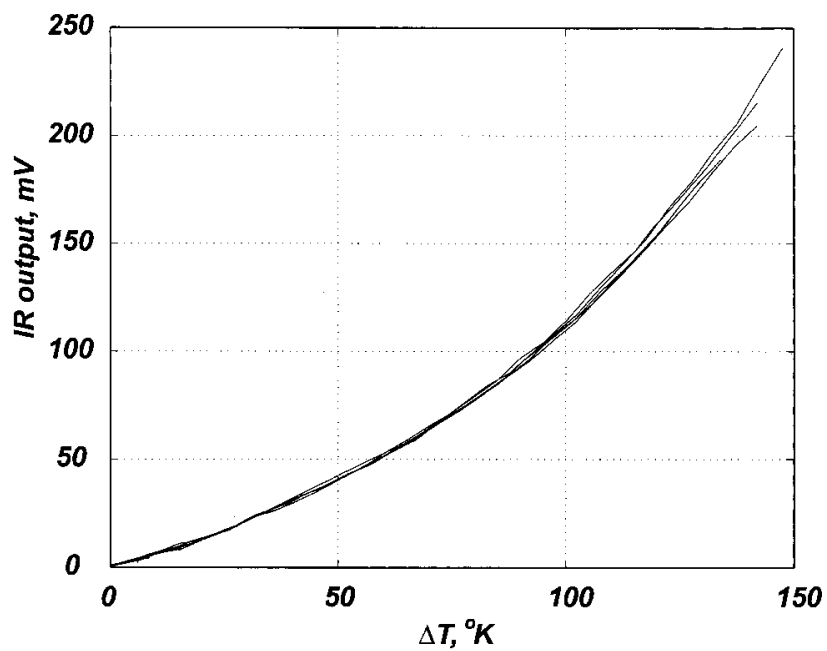

FIG. 7. Calibration of IR detectors using $1.3 \times$ lens system and C-300 steel sample. Each curve in the plot corresponds to a repeat of the calibration.

placed in the object position and heated with an electrical current. Using the $1.3 \times$ lens the $75 \mu \mathrm{m}$ wire gets mapped to $100 \mu \mathrm{m}$ on the focal plane array. If the focus were perfect and if there were no cross talk between the detector elements, once the system is focused to obtain the maximum signal on a single column of elements, there should be no signal on the adjacent elements. In practice there is electrical cross talk between elements and the focusing is not perfect. At the best focus, adjacent elements output a signal that is $15 \%$ of the element the hot wire is focused on. The next nearest neighbors output a signal that is $6 \%$. The crosstalk check is repeated with the wire in the horizontal position. Knowing this crosstalk we can correct for it in the analysis of the data by setting up and solving 64 linear equations of the form

$$
\begin{aligned}
s_{i}=\frac{1}{1.84}[ & r_{i}+0.15\left(r_{i+1}+r_{i-1}+r_{i-8}+r_{i+8}\right) \\
& \left.+0.06\left(r_{i+2}+r_{i-2}+r_{i-16}+r_{i+16}\right)\right],
\end{aligned}
$$

where the elements are numbered $i=1,64, s_{i}$ is the measured signal from element $i$, and $r_{i}$ is the actual signal on element $i$. The $1 / 1.84$ term is there to give $r_{i}=s_{i}$ when there is uniform illumination. These equations are modified for elements at the edges of the array since they do not have neighbors to one side.

\section{B. Temporal resolution}

The temporal resolution is limited by the bandwidth of the detector-amplifier system and the $1 \mathrm{MHz}$ maximum digitization rate. To verify the time response of the system, an IR emitting light emitting diode (LED) is placed in front of the $\mathrm{HgCdTe}$ detector array and pulsed. The rise time in response to a step input from the IR LED is approximately $1 \mu \mathrm{s}$, equivalent to a $500 \mathrm{kHz}$ bandwidth.

\section{Calibration}

Calibration of the system is performed in a very direct manner. A sample of the same material and surface finish as will be tested is heated in an oven to several hundred degrees

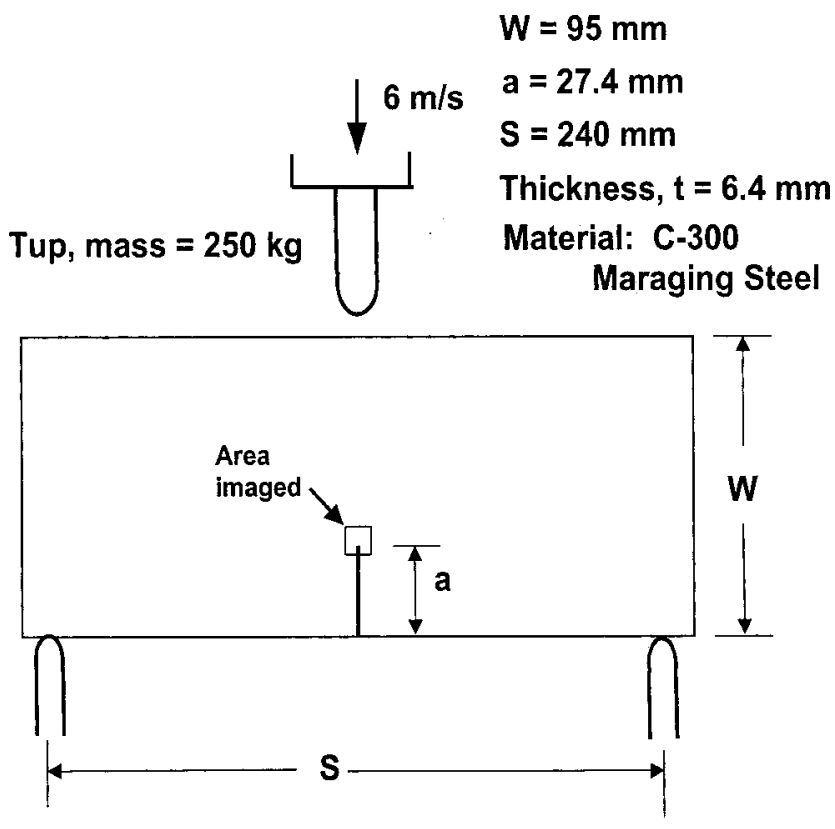

FIG. 8. Schematic of notched C-300 steel drop weight impact experiment.

kelvin. The heated sample, instrumented with a thermocouple to record its surface temperature is placed in the object position. As the sample cools, the voltage output from each of the IR elements is recorded by the data acquisition system along with the sample temperature. This procedure provides a voltage versus temperature curve for each element.

Since the IR system is ac coupled, the input radiation to the detectors must contain an ac component. This is achieved by placing a chopping wheel in front of the heated calibration specimen. A LED-photodiode pair is placed across the chopping wheel to synchronize the data acquisition with the chopping. While the chopping wheel is covering the calibration sample, 64 points are recorded. Another 64 points are recorded while the wheel exposes the sample. Each of the sets of 64 points is averaged, then their difference taken to provide the peak-to-peak voltage output corresponding to a given temperature. A typical calibration curve, obtained using the $1.3 \times$ magnification lens is shown in Fig. 7. Near room temperature, the sensitivity is approximately $1 \mathrm{mV} / \mathrm{K}$. We have found that there are significant variations in the outputs of the detector elements, thus each element must have its own calibration. The system noise level, which, as recorded by the $\mathrm{A} / \mathrm{D}$ boards, is approximately $2 \mathrm{mV}$, corresponding to a temperature resolution of $2 \mathrm{~K}$. The thermal resolution can be increased by using lower magnification, causing each detector to gather radiation from a larger area. The scatter in the calibration determines the uncertainty in the temperatures. For the $1.3 \times$ system calibration plotted in Fig. 7 the uncertainty in temperature is approximately $\pm 5 \%$.

\section{RESULTS}

One of the applications of the system was to characterize the transient temperature fields associated with the process of crack initiation and growth in a high strength steel. A C-300 maraging steel plate (yield strength of about $1900 \mathrm{MPa}$ ) with 

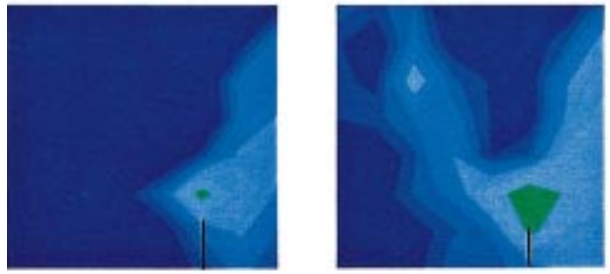

$$
t=170 \mu s
$$

$178 \mu s$

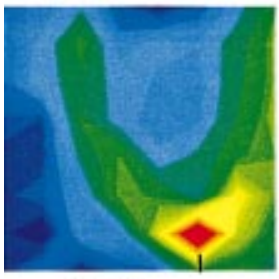

$202 \mu s$

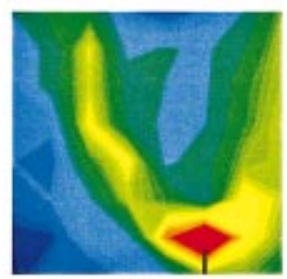

$210 \mu s$
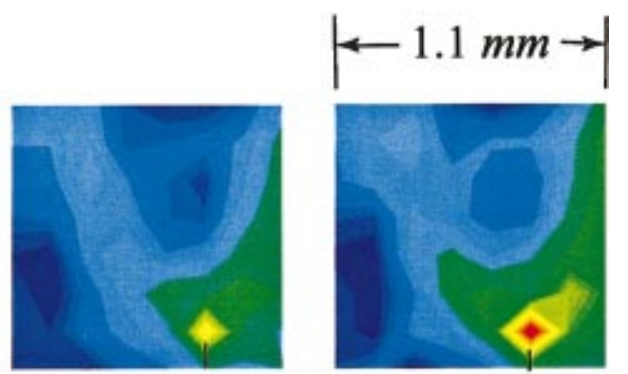

$186 \mu \mathrm{s}$

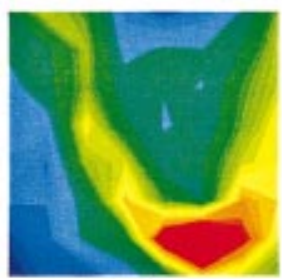

$218 \mu s$

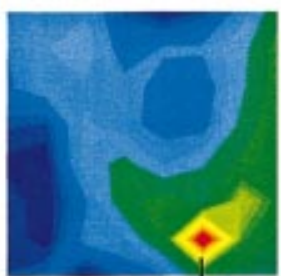

$194 \mu s$

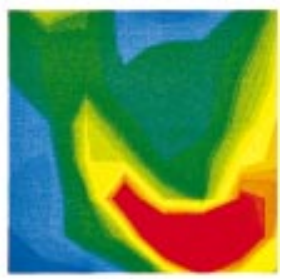

$226 \mu \mathrm{s}$

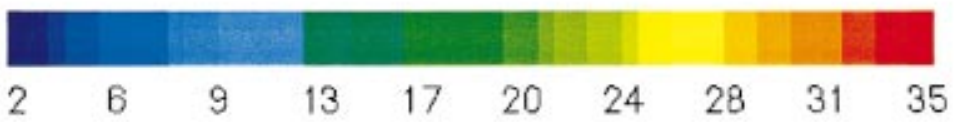

(a)

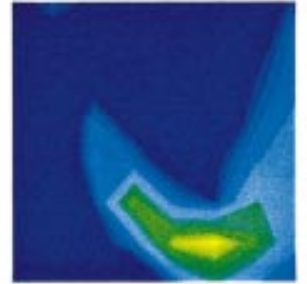

$t=226 \mu s$

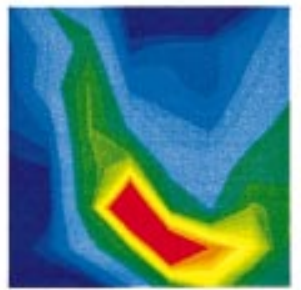

$234 \mu s$

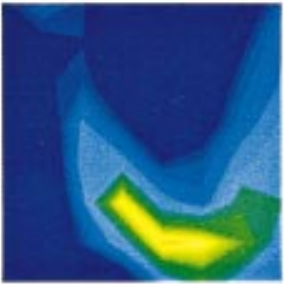

$228 \mu s$

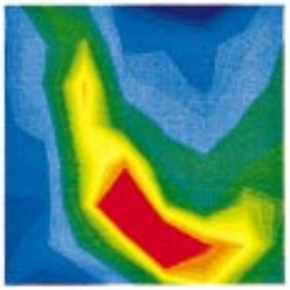

$236 \mu s$
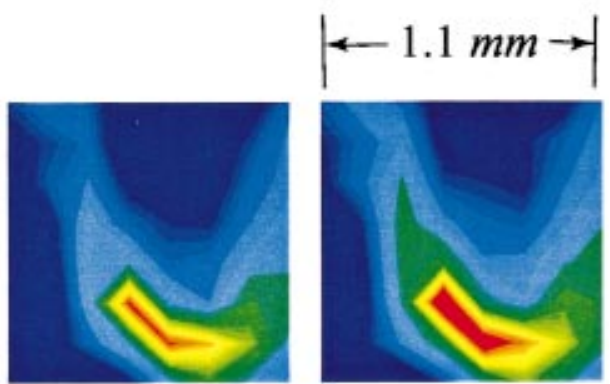

$230 \mu s$

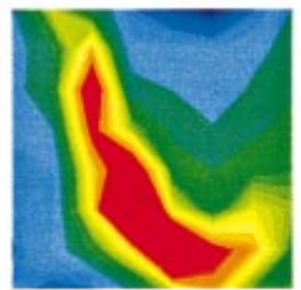

$238 \mu s$
FIG. 9. Temperature field for crack initiation (color image). (a) Temperature field during loading (prior to crack initiation). (b) Temperature after crack begins to grow. Peak temperatures move with crack tip. Note that temperature contours are linear interpolations.

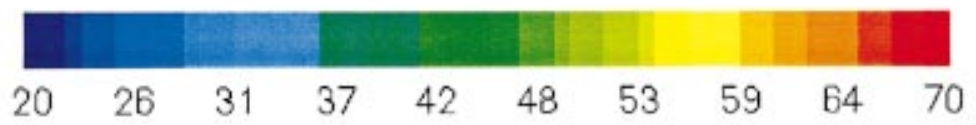

(b)

a prefabricated crack, was impact loaded in a drop weight tower as shown in Fig. 8. The IR imaging system was focused on an area of approximately $1.1 \times 1.1 \mathrm{~mm}$, as shown in the figure.
Two sequences of thermal images form the same experiment are shown in Fig. 9. Note that the last image in Fig. 9(a) is the same as the first image of Fig. 9(b). They appear different because of the different temperature scales used in 
plotting them. In Fig. 9(a), a more or less symmetric, "U', shaped zone of high temperature develops at the crack tip about $160 \mu$ s after impact. This zone of high temperature corresponds to the region in which plastic deformation occurs. Thus, the thermal images show us that the deformation is somewhat localized along diagonal bands emanating from the crack tip. The temperature field stays symmetric until about $226 \mu \mathrm{s}$.

Asymmetry in the temperature distribution can be seen to begin and build up rapidly in Fig. 9(b). This is an indication that the crack has begun to grow. This experiment provides us the detailed distribution of the temperature field around the crack tip and an indication of the associated distribution of dissipated plastic work at the specimen surface. Taking the crack initiation time to be around $226 \mu$ s, the experiment also revealed that the specimen surface at the crack tip heats up by as much as $55 \mathrm{~K}$ by the time of crack initiation. This temperature rise under different loading rates could be used as a measure of the resistance of the material to crack growth. The observed temperature distribution could also serve as a bench mark for comparing any future theoretical/computational studies on crack initiation and propagation in similar materials.

By using a fully row-parallel data acquisition scheme, whereby data from each row of an IR focal plane array is acquired simultaneously, a system capable of acquiring $8 \times 8$ IR images at a rate of one million frames per second has been assembled from a combination of standard and custom built components. This system, consisting of an $8 \times 8$ $\mathrm{HgCdTe}$ focal plane array, 64 preamplifiers and track and hold amplifiers, eight 8:1 multiplexers, eight $10 \mathrm{MHz}, 12$ bit digitizers, and a fully reflective lens system, is capable of spatial resolution down to approximately $50 \mu \mathrm{m}$, and a temperature resolution of approximately $2 \mathrm{~K}$. The system has been used to image the distribution of temperature rise near the tip of a notch in a high strength steel sample (C-300) subject to impact loading by a drop weight testing machine. The results show temperature rises at the crack tip up to around $70 \mathrm{~K}$. Localization of temperature, and hence, of deformation into $\mathrm{U}$ shaped zones emanating from the notch tip is clearly seen, as is the onset of crack propagation. Many other applications of this system to solid and fluid mechanics can be anticipated. A few examples include: temperatures in reactive flows, temperature rise in dynamic shear banding, and temperature rise during ballistic impact.

\section{ACKNOWLEDGMENTS}

This work was performed at Caltech with support from the National Science Foundation, through Grant No. CTS9512517, the Department of Energy, Grant No. DE-FG0395-ER14560, and the Office of Naval Research, Grant No. N00014-95-1-0453. The design and most of the fabrication of this system were performed while the first author was a sabbatic visitor to Caltech in the 1996-97 academic year. The authors would like to thank Fermionics Inc. for custom building the IR detector array and Gage Applied Sciences for a substantial discount on the digitizing system.

${ }^{1}$ A. T. Zehnder and A. J. Rosakis, J. Mech. Phys. Solids 39, 385 (1991)

${ }^{2}$ J. A. Kallivayalil and A. T. Zehnder, Int. J. Fract. 66, 99 (1994).

${ }^{3}$ A. T. Zehnder and A. J. Rosakis, in Experimental Techniques in Fracture, edited by J. S. Epstein (VCH, New York, 1993), Vol. III, p. 125.

${ }^{4}$ H. Ay and W.-J. Yang, Int. J. Heat Mass Transf. 41, 613 (1998).

${ }^{5}$ M. Zhou, A. J. Rosakis, and G. Ravichandran, J. Mech. Phys. Solids 44, 981 (1996).

${ }^{6}$ K. A. Hartley, J. Duffy, and R. H. Hawley, J. Mech. Phys. Solids 35, 283 (1987).

${ }^{7}$ G. T. Camacho and M. Ortiz, Comput. Methods Appl. Mech. Eng. 142, 269 (1997).

${ }^{8}$ Y. Wang, Ph.D. thesis, Wayne St. University, 1997.

${ }^{9}$ R. Weichert and K. Schoenert, J. Mech. Phys. Solids 26, 151 (1978).

${ }^{10}$ J. Hodowany, G. Ravichandran, A. J. Rosakis, and P. Rosakis, GALCIT SM Report 98-7, California Institute of Technology, Pasadena, CA, 1998.

${ }^{11}$ R. Kingslake, Lens Design Fundamentals (Academic, New York, 1978).

${ }^{12}$ Rolyn Optics, Covina, CA.

${ }^{13}$ Fermionics, Simi Valley, CA. 